\title{
The Edinburgh Lithium Register
}

\author{
Robert E. Kehoe, Registrar in Psychiatry; and ANThONy J. MANDER, Lecturer, \\ University of Edinburgh, Royal Edinburgh Hospital, Morningside Terrace, Edinburgh \\ EH10 5HF
}

Lithium is widely used in psychiatry, mainly in the prophylaxis of manic-depressive illness. It is usually prescribed long term and because of its side effects, low therapeutic index and the possibility of toxicity, it is necessary to monitor serum lithium levels, thyroid function and renal status. Clinical guidelines are unclear; Vestergaard et al (1982) recommended that serum lithium and creatinine be checked every two to four months and thyroid function every six months. However, Crammer et al (1982) suggest that once stable levels are established, serum lithium need be checked only in response to clinical indications. The most recent edition of a widely read psychiatric text states that levels should be checked each six to eight weeks for as long as the patient continues on lithium (Loudon, 1988). Accepted standard clinical practice is thus unclear. It should be emphasised that these guidelines are not based on research evidence.

It is widely accepted that toxic effects are minimised if lithium levels are maintained within the range $0.5-1.0 \mathrm{mmol} / 1$. In Edinburgh serum lithium concentration is checked every six to twelve weeks once stable levels have been established and renal and thyroid functions are measured every six to twelve months. The Edinburgh Lithium Register reminds practitioners to check these parameters and hence provides an inexpensive way of supervising lithium prophylaxis.

\section{The Register}

This is shown as a flow diagram (Figure 1).

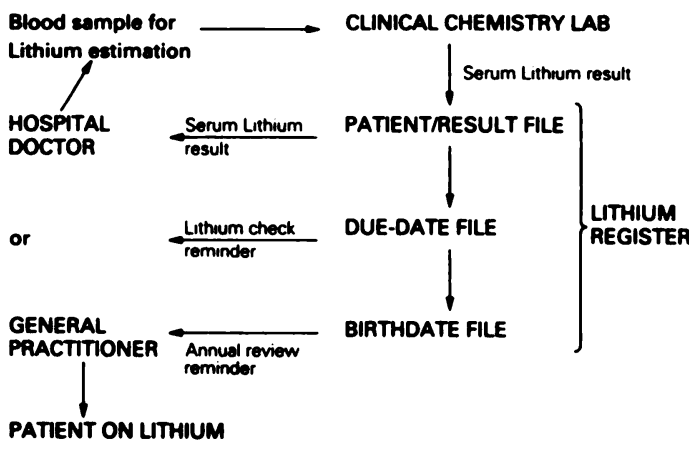

Fig. 1. Lithium Register flow diagram.

\section{(1) Samples and results}

Serum lithium levels for patients living in Edinburgh and the surrounding Lothian Region are measured by only one NHS laboratory at the Clinical Chemistry Department in the Royal Infirmary. Results are sent to the Lithium Register Secretary at the Royal Edinburgh Hospital, the major psychiatric teaching hospital, before being dispatched to the requesting practitioner - both GP and hospital based.

\section{(2) Card files}

\section{(a) Alphabetic index by surname}

This is a longitudinal record of lithium results. On the front of each card demographic details are recorded - name, address, date of birth, GP, hospital practitioner and hospital unit number (if any). On the reverse, the date and result of each lithium check is recorded in columns-and the date 12 weeks hence is recorded adjacent to the result. After this information has been recorded, the lithium result is forwarded to the requesting practitioner.

\section{(b) 'Due-date' file}

This is the basis for sending out reminders to check lithium levels.

At the same time as completing the results in card (a), a card for each patient is filed in a separate index system under the appropriate date for 12 weeks in the future (one date for the beginning of each week). If, in the next 12 weeks a further lithium estimation is received, this card is retrieved and filed again under the date for 12 weeks in the future. If no lithium result is received by the 'due-date' then that card and others under the 'due-date' are pulled out and a reminder is posted to the practitioners stating that their patient is overdue for a lithium check. They are also asked to inform the office if the patient is no longer taking lithium.

\section{(c) Birthdate file}

This is used for notification of annual review of long-term lithium therapy.

A card for each patient is filed by their date of birth in this calendar file. Once a month letters are sent out 
to practitioners reminding them of the need for an annual review of those patients whose birthdates fall in that month. They are asked to consider checks of thyroid function and renal creatinine clearance and also to check for clinical signs suggestive of cardiovascular disease. It may be the case that the practitioner has already performed these investigations.

\section{(3) Costs}

(a) Personnel-currently 15 hours per week of secretarial time; approximately $£ 2,500$ per year.

(b) Postage for reminders and stationary-both costs are minimal as forms are already being sent in the post.

\section{Discussion}

The lithium register provides a simple method of lithium surveillance. Hence a traditional lithium clinic where patients attend the hospital to have their levels checked is unnecessary. Patients benefit from the convenience of having samples taken by their general practitioner. The hospital benefits from the decreased costs, which could be reduced further by using an assistant trained in the use of the register rather than a highly qualified medical secretary. Finally, general practitioners have the satisfaction of retaining responsibility for their patients' care with the back-up of regular reminders from the hospital. The register causes a delay of approximately 24 hours in the result being received by the requesting doctor. For the vast majority of patients this is unimportant and urgent requests and levels above the therapeutic range are communicated directly to the doctor by the laboratory staff over the telephone.

This system enables us to define the number of people being monitored for lithium treatment within the region and gives information regarding the duration of such treatment and the frequency of monitoring. There is, however, little knowledge as to what extent practitioners respond to the 'reminders' and thus a 'feedback loop' is lacking. This applies to annual reviews as well as to actual lithium level monitoring. In earlier years, practitioners were contacted by letter or telephone when there was no response to lithium-level reminders. This would act as a further reminder or record if the patient had discontinued lithium or moved to a different area.

The lithium register is now well established and has been in use since 1970 . It could be improved by increasing the feedback about thyroid and renal function, as well as to the response to reminders for lithium checks. Computerisation might also increase efficiency; an attempt to run a computer-based system in the early 1980 s failed through lack of finance. The publication of Working for Patients with its emphasis on audit and new information technology might enable successful computerisation in the near future. If, as the Government predicts, substantial numbers of GPs invest in their own computer systems then a hospital-GP link would be feasible. Part of the function of such a system could be to update practitioners on current guidelines for lithium monitoring as well as to improve the stringency of clinical practice and audit. The register also provides an excellent data base for research.

We are currently involved in an audit of the system designed to ascertain how often patients have their lithium levels checked, how quickly abnormal results are corrected and whether there are any differences between GP and hospital monitored patients. The results of these studies should enable further improvements to be made to the efficiency of lithium monitoring in this region.

\section{References}

Crammer, J., Barraclough, B. \& Heine, B. (1982) The Use of Drugs in Psychiatry. London: Gaskell (Royal College of Psychiatrists).

Loudon, J. B. (1988) Drug treatments. In Companion to Psychiatric Studies (eds. R. E. Kendell and A. K. Zealley). Edinburgh: Churchill Livingstone.

DHSS (1989) Working for Patients. London: HMSO.

Vestergand, P., Shou, M. \& Thomsen, K. (1982) Monitoring of patients in prophylactic lithium treatment an assessment based on recent kidney studies. British Journal of Psychiatry, 140, 185-187. 